\title{
Assessment of the Environmental Impact of Modern Dry Cargo Ships
}

\author{
Ivan Karpushin ${ }^{1}$, Vitalii Gannesen ${ }^{1}$, and Ekaterina Solovyeva ${ }^{1 *}$ \\ ${ }^{1}$ Far Eastern State Technical Fisheries University, 52bLugovaya Str., Vladivostok, 690087, Russia
}

\begin{abstract}
The article deals with the impact of dry cargo ships on the environment. The transportation of bulk cargo takes a significant part in the total transportation of goods today, and a significant part of the cargo is transported by universal dry cargo ships.
\end{abstract}

\section{Introduction}

About 9.5 billion tons of cargo are transported across the oceans annually, and bulk cargoes make up the largest group of cargoes. Discharge of cargo residues after unloading bulk carriers into the sea which occurs on a regular basis poses a threat of pollution.

More than 2.15 million tons of bulk cargo is reported to be entering the oceans of which 100,000 tons are potentially harmful to the marine environment.

The purpose of the work performed is to study the impact of the bulk cargo discharge on the environment.

To achieve this goal, the following issues were considered: procedures for bulk cargo receiving and processing, control over bulk cargo loading, transportation of dangerous goods, checking cargo securing. The work was done on the example of M/V Prosper in accordance with the recommendations and restrictions available in the specifications of the vessel.

Sea transport is the backbone of the world economics. It is generally accepted that over $90 \%$ of world trade cargo is delivered by sea. In terms of volumes, bulk cargo is the largest group which constitutes more than $50 \%$ of all shipments.

\section{Key research findings}

Delivery of bulk cargo is fraught with dangers that may arise during transportation due to the physical and chemical properties of these cargoes.

According to worldwide statistics, a certain number of ships carrying bulk cargo including new specialized ships wreck each year.

The International Maritime Organization (IMO) after a detailed review of the reasons for the loss of ships has summarized the available experience and adopted a number of international documents regulating the safe transportation of goods such as SOLAS 74, MARPOL 73/78, and the Code of Safe Practice for the Carriage of Bulk Cargoes. $[1,2,3]$.

*Corresponding author: soloveva.ee@dgtru.ru 
It should be noted that the Code is of a recommendatory nature, but in some countries the national regulations for delivery of non-grain bulk cargo are being developed on its basis. In some countries, the Code is applied as the national regulations [11].

Discharge of ship's waste in the oceans is governed by the requirements of the International Convention for the Prevention of Pollution from Ships, 1973, as amended by the Protocol of 1978 (Convention MARPOL 73/78) taking into account the amendments to the Convention Annexes I, II, IV and V approved by the 68th session of the IMO Marine Environment Protection Committee (MEPC) in May 2015 [1].

At the moment MARPOL incorporates six annexes dealing with the prevention of several types of pollution. For various reasons, no separate annex for bulk cargo has been drawn up, but it is believed that the garbage dumping rules (specified in Appendix V) are applied which aim to zero pollution and restrict any garbage dumping. However, during discussions at IMO it was noted that although prohibited it is a common practice to wash overboard the remains of cargo left on bulk carriers after unloading. According to information from shippers, these "residues" could represent the discharge of 60 and more tons of cleaning solution per hold during usual operations on board.

For this reason, IMO considered necessary to establish the clearer rules at the regulatory level and revised MARPOL Annex $\mathrm{V}$ to better deal with the potential environmental impacts of bulk cargo [1].

In accordance with the amendment to the Convention MARPOL Annex V in 2012 it has been prohibited to discharge cargo residues harmful to the marine environment since 2015.

The International Convention for the Safety of Life at Sea 1960 (SOLAS Convention) contained the mandatory provisions in parts A and B of Chapter VI and in part A-1 of Chapter VII governing the carriage of bulk cargo and the carriage of dangerous goods in bulk, respectively. The Convention for the Safety of Life at Sea 1974 entered into force on 25 May 1980, replaced the 1960 Convention, and in 1983 the Maritime Safety Committee adopted a revised Chapter VII. The amendments to this Chapter entered into force on 1 July 1986. Currently Section A of Chapter VII regulates delivery of dangerous goods, both packaged and solid bulk [2].

The problems associated with the transportation of bulk cargo were recognized by the delegates of the 1960 International Conference on the Protection of Human Life at Sea, but at that time it was impossible to develop detailed requirements, except for the requirements concerning the transportation of grain. The Conference, however, recommended in paragraph 55 of Annex D to the Convention that an internationally acceptable Code of Safe Practice for the Transport of Bulk Cargo be developed under the auspices of the International Maritime Organization (IMO). This work was undertaken by the Subcommittee on Containers and Cargo of the Organization, and since the first publication in 1965 of the Code of Safe Practice for the Transportation of Bulk Cargo (NG Code), several editions of this Code have been issued [3].

The International Sea Bulk Cargo Code (IMSBC) which became mandatory on 1 January 2011 in accordance with the SOLAS Convention is the main regulation governing the safe delivery of bulk cargo.

IMO Resolution MSC.393 (95) amended (03-15) the International Sea Bulk Cargo Code (IMSBC). The amendments became mandatory on 1 January 2017.

IMO Resolution MSC.426 (98) amended (04-17) the International Marine Bulk Cargo Code (IMSBC). The amendments became mandatory on 1 January 2019.

IMO Resolution MSC.462 (101) amended (05-19) the International Marine Bulk Cargo Code (IMSBC). The amendments become mandatory from January 1, 2021 and can be introduced voluntarily from 1 January $2020[4,5]$.

The International Maritime Bulk Carriage Code (IMSBC) approved by the Maritime Organization's Safety Committee resolution MSC.268 (85) is a guideline recommended for 
use by Administrations, ship owners, shippers and masters, and provides standards for the safe stowage and delivery of solid bulk cargoes with the exception of grain the carriage of which is regulated by separate rules.

The Code contains general guidelines that should be followed whenever bulk cargo is delivered by sea, a description of the hazards specific to certain materials, lists of typical substances currently carried in bulk, and a detailed description of recommended methodologies for determining the various characteristics of bulk substances $[4,5,9,10]$.

It should be noted that the list of substances set forth in Annexes A, B and C to the Code is not an exhaustive one, and the physical properties of the substances are given only to get a general idea. As a result, before loading any bulk cargo it is necessary to obtain updated information on its physical and chemical properties from the consignor.

The main purpose of the Code is to promote the safe loading and shipment of solid bulk cargoes by providing information on the hazards associated with the delivery of certain types of cargo and on the standards to be applied for the transportation of solid bulk cargoes, excluding grain $[8,9,10]$.

The Code lists the types of cargo that are shipped in bulk and provides instructions on appropriate safety procedures (storage requirements, maximum moisture content, etc.) $[4,5]$.

The most part of the Code is devoted to the provisions of Appendix 1 Individual Bulk Cargoes which provides an alphabetical listing of cargoes usually shipped in bulk. Each cargo is described in detail, including its characteristics (eg. angle of repose, stacking ratio and group $\mathrm{A}, \mathrm{B}$ or $\mathrm{C}$ ). It should be noted that the group to which the product belongs provides important safety information as defined in the IMSBC Code:

- Group A consists of cargoes that are subject to liquefaction;

- Group B consists of cargoes that have a chemical hazard;

- Group C - cargoes that are not liable to liquefaction (Group A) and do not have a chemical hazard (Group B).

A significant problem is that not all bulk cargoes are currently listed in the IMSBC Code. In addition, it has been found that there are a number of goods designated in group $C$ that can demonstrate the characteristics of goods in groups A and B.

The IMSBC Code is regularly updated as more information on the properties and potential hazards of bulk cargoes becomes available through industry research $[9,10]$. This process continues. Thus, the Code is still under development and by itself cannot provide a completely reliable barrier to the hazards associated with bulk cargo.

The estimated annual operational maritime production of 2-3 million tons of solid bulk cargo (including some 80,000 tons of heavy cargo) is significantly higher than the estimated 460,000 tons of oil to the marine environment from ships.

Dumped cargo residues may concentrate in certain sea areas along major shipping routes after leaving ports of discharge which are only permitted outside the 12 nautical miles offshore zone.

In 2016 it was decided that the classification criteria and a declaration by the shipper of bulk cargoes whether they are harmful to the marine environment should become mandatory in the future.

The criteria seem to be reasonable ones as they address environmental issues such as ecotoxicity (criteria 1 and 2), contamination (criterion 7), and health risks from seafood consumption (criteria 3-6). They are similar to the criteria used for bulk cargoes regulated by MARPOL Annex II. [1]

However, the direct application of these criteria as hazard indicators does not appear to be suitable for bulk cargoes as they were mainly developed for well-defined organic chemicals, whereas most bulk materials that are potentially harmful to the marine environment, are mineral ores and metal concentrates with variable compositions and physicochemical characteristics. 
The latest amendment to the IMSBC Code was provided by IMO in Resolution MSC.462 (101), adopted on 13 June 2019. It was agreed that IMO flag State members could elect to apply these amendments from 1 January 2020 and that they will come into force on a mandatory basis from 1 January 2021 [5].

The current rules for bulk cargo shipment require shippers to classify whether a particular cargo meets the criteria «harmful to the environment» (HME). However, this classification suffers not only from the ambiguity just explained, but also from the lack of available information on the chemical composition, bioavailability and bioaccumulation of specific components of the shipped consignments.

It can be doubted whether MARPOL Annex V dealing with garbage is the best rule for dealing with the issue of dumping cargo residues.

Special rules for cargo residues similar to the rules of Annex II to MARPOL for bulk cargo could be more appropriate, since very specific handling characteristics could be better taken into account (for example, the definition of specific unloading requirements or maximum unloading volumes in accordance with the cargo specification) $[3,5,8]$.

Solid bulk cargoes shall be classified and declared by the consignor as posing a threat to the marine environment or not, in accordance with the criteria set out in paragraph 3.2 in 11 of the 2012 Guidelines for the Implementation of MARPOL Annex V [1].

\section{Conclusion}

Based on above said, it can be concluded that bulk cargo shipped by bulk carriers can enter the marine environment at different stages of deliveryn: loading, transshipment, unloading and washing of cargo holds.

Any type of risk assessment requires hazard and exposure information (or at least emission data as a surrogate). Due to the identified difficulties in assessing the hazardous properties of bulk cargo and uncertainties related to impact, a comprehensive assessment of environmental risk is currently unrealistic.

The article is published within the "Development of a Competitiveness Enhancement Methods of Fishing Fleet" (No.121031300159-6) government program of the Federal Agency for Fishery.

\section{References}

1. International Convention for Prevention of Pollution from Ships (MARPOL-73/78). $\mathrm{SPb}$.: ZAO TsNIIMF, 412 (2017)

2. International Convention for the Safety of Life at Sea, 1974, (SOLAS-74). (Text modified by the Protocol of 1988 relating thereto, including Amendments). SPb .: ZAO TsNIIMF, 1184 (2021)

3. International Maritime Solid Bulk Cargoes Code (IMSBC Code), 1096 (2012)

4. International Code for the Carriage of Bulk Cargoes by Sea (ICMPNG). Bulletin of amendments and additions No. 1- SPb .: ZAO TsNIIMF, 81 (2012)

5. IMDG Code International Maritime Dangerous Code, vol. 1-2, 2020 Ed. (inc.Amdt. 40-20), vol. 1-2

6. IMDG Code Supplement 2020 (Ems Guide, MFAG Guide), 2020.

7. Bulletin No. 40 of amendments and additions to the International Convention for the Safety of Life at Sea 1974 (MK SOLAS-74) ed. 2021 - St. Petersburg: AO TsNIIMF, $60(2021)$ 
8. Code of Practice for the Safe Loading and Unloading of Bulk Carriers (BLU Code), 80 (2011)

9. IMDG Code International Maritime Dangerous Code, vol. 1-2, 2018 Ed. (inc.Amdt. 39-18), vol. 1-2.

10. IMDG Code Supplement 2018 (Ems Guide, MFAG Guide).

11. V.I. Dmitriev, E.V. Naydenov, Transportation of goods by sea, Morkniga, 343 (2021) 